partitioned between the tissue and the extracting solution. The equilibrium concentration of pectin methylesterase in solution was altered by the addition of auxin. Presumably the growth of the plant requires that pectin methylesterase be attached to the wall tissue, and the major action of the auxin is that it should be able to reach the appropriate tissue and cause adsorption of pectin methylesterase and perhaps other enzymes.

The group also investigated the movement of sugars into the storage tissues of cane. The uptake was shown to consist of two processes. The first process was that of diffusion into the outer space of the tissue volume, and was governed by the concentration gradient, equilibrium being attained in about half an hour. The second process continued at a constant rate for about $60 \mathrm{hr}$. and sugar moved against a considerable concentration gradient. The movement was prevented by anaerobic conditions and a number of metabolic inhibitors. This active uptake of sugar into the inner space is analogous to the accumulation of salts by other plant tissues. It was shown that only sucrose-and not reducing sugars-could be taken into the inner space, but that loss from this space was mainly glucose or fructose formed by hydrolysis, particularly in immature tissue. The turnover time for the sucrose pool for young tissue was about $9 \mathrm{hr}$.

To make use of these observations and other fundamental data, detailed studies are required of the effects of the environment on growth and the translocation and accumulation of sugars. Comparative studies will be made on different species and varieties within species of the genus Saccharum. The central interest will be the ability to store carbohydrate as sucrose.
The genetic make-up of commercial varieties of sugar cane is derived from $S$. officinarum, but virtually all varieties now cultivated carry genes from other species, usually with very low sugar content. Hybridization was carried out to increase vigour, resistance to disease, and to widen the range of climatic conditions in which cane can be cultivated successfully. It is anticipated that the comparative studies on sugar accumulation will provide knowledge of the mechanisms by which canes do or do not store sucrose, and this information can be used as a basis for studies on the physiological and biochemical genetics of sugar production.

The work at the David North Plant Research Centre will also include a comprehensive investigation on flowering in the genus Saccharum and several related genera and on problems associated with sterility and with the germination of its seeds. Complementing this programme, more general studies will be undertaken in the field of phytohormonal and chemical control of plant growth.

The research staff will consist of five graduates and assistants. Space will be made available for two or three guest research workers from other institutions to work with the Colonial Sugar Refining Co.'s group.

Other laboratories situated in Brisbane which carry out research on plants are located in several departments of the University of Queensland, at the Cunningham Laboratory of the Commonwealth Scientific and Industrial Research Organization and the laboratories of the Queensland Sugar Bureau. This concentration of research groups should establish Brisbane as a leading centre for research on tropical plants in the southern hemisphere.

K. T. Glasziou

\title{
SOME ASPECTS OF AUTOXIDATION
}

A $\mathrm{N}$ informal symposium on the "Physico-chemical, Biological and Applied Aspects of Autoxidation" was held during April 4-5 in the Department of Inorganic and Physical Chemistry of the University of Liverpool. The meeting was organized by Dr. N. Uri (Ministry of Agriculture, Fisheries and Food Research Establishment, Aberdeen) on behalf of the Colloid and Biophysics Committee of the Faraday Society, and its host, Prof. C. E. H. Bawn, was its chairman.

On the afternoon of the first day four papers, dealing mainly with physico-chemical aspects, were presented to the meeting. Prof. J. C. Robb (University of Birmingham) spoke about a new method in work on autoxidation of hydrocarbons in the liquid phase. He explained the considerable experimental difficulties that are encountered when the rates of diffusion of oxygen from the gaseous into the liquid phase must be taken into account. A method has been developed in which the oxygen already dissolved is used up during kinetic measurements without the necessity for replenishment. The rate of reaction is followed by the measurement of rise in temperature by means of a thermocouple and a sensitive amplifier. A rise of $10^{-3} \mathrm{deg}$. C./sec. can be determined with a time response of about $10^{-2}$ sec. The sensitivity of the method enables one to study non-stationary build-up of radical concentration and to obtain quantitative information on rate constants of propagation and termination as well as reactivity of free radicals towards retarders. Autoxidation of cyclohexene and methyl cyclohexene have been studied at temperatures of $40-80^{\circ} \mathrm{C}$.

The second paper, which dealt with the initiation phase in the aerobic oxidation of linoleie acid and its esters, was presented by Dr. N. Uri. Evidence was presented suggesting that metal catalysts are important even in the apparently uncatalysed autoxidation. A survey of the effects of heavy-metal stearates and porphyrins indicated that there must be a complex system of initiation involving oxygen-metal complex formation and subsequent formation of free radicals. A reaction scheme was proposed which involved reactions of the metal compound with oxygen, hydroperoxide and substrate and included an inhibitory reaction between metal and free radicals; the latter feature explained an anomalous curve obtained when the rate of reaction was plotted against metal catalyst concentration. Some experiments relating to the effects of environment and chelating on the catalytic activity of the initiaton were described. A brief survey of the effects or various novel free radical acceptors and their theoretical interpretation concluded the paper. If 
the discussion Dr. Ford (British Petroleum, Sunbury) directed attention to the fact that anomalous initiation curves were observed by him when ham pigments were used as autoxidation catalysts.

Prof. C. E. H. Bawn introduced his paper on the metal-catalysed oxidation of alkyl aromatics by emphasizing the practical significance of this work in the synthesis of new products and in the deleterious effect causing break-up and degradation of oils and plastics. The oxidation of isopropylbenzene to phenol and of $p$-xylene to terephthalic acid are important examples. Recent discoveries have shown that the first stage of the oxidation of $p$-xylene, that is to say, to $p$-toluic acid, proceeds satisfactorily with the conventional cobalt and manganese catalysts, but the second stage requires the presence of bromide. Detailed kinetic results were presented, and a satisfactory mechanism involving initiation by the bromine atom in conjunction with the cobaltous-cobaltic cycle, was proposed. Inhibition of the second oxidation reaction in the absence of added bromine was shown to be associated with the formation of phenolic inhibitors which are destroyed by bromine. In the discussion, Dr. Uri pointed out that metal-catalysed initiation in the oxidation of poly-unsaturated fatty acids is more closely related to that generally proposed by Prof. Bawn for aldehydes rather than that proposed for hydrocarbons. In reply to Prof. H. S. Olcott (University of California, Berkeley), Prof. Bawn explained that iodide could not replace bromide. Dr. C. H. Lea directed attention to various salt effects in the oxidative rancidity of fats which are not yet fully explained.

The fourth paper, by Dr. L. Bateman (British Rubber Producers' Research Association, W elwyn), dealt with the oxidation and antioxidant activity of organic sulphur compounds related to rubber vulcanizates. $\mathrm{He}$ emphasized that the response of these complex organic sulphides to atmospheric oxidation was not yet fully understood although it was apparent that it differed considerably from that of the parent hydrocarbon polymer. There are some distinctive features: the reaction is catalysed photolytically, by free-radical generators such as azoisobutyronitrile, but not by peroxides ; $\alpha$-hydroperoxysulphide initially formed is converted rapidly into sulphoxide and $\alpha$-hydrosulphide and the reaction is subject to autoretardation. It was shown that sulphoxides formed from monosulphides and thiosulphinates formed from disulphides were efficient inhibitors of olefin oxidation. The effects of substituents on the efficiency of these inhibitors were discussed in detoil, and stross was laid on the practical significance of these results in the interpretation of the oxidation characteristics associated with different vulcanizates.

On the second day of the meeting, Dr. J. Friend (Low Tomperature Research Station, Cambridge) spoke about some aspects of the biologically catalysed aerobic oxidation of carotenoids. The nature of the enzyme systems responsible for the carotenoid destruction in plant tissue was discussed. These include lipoxidase and lipoperoxidase (rocently studied by Dr. Blain) in seedlings and different enzyme systems in chloroplasts and mitochondria prepared from sugar beet leaves; the latier do not require the presenco of linoleic acid or diene hydroperoxide. A comparison of the degradation products by enzymic oxidation and non-enzymic oxidation catalysed by linoleate and ferrous phthalocyanine revealed close similarity.
In a paper on the aerobic oxidation of plant phenols and its biological implications, Dr. D. E. Hathway (British Leather Manufacturers Research Associntion, Egham), exemplified the synthesis of larger molecules from simple phenols by dimerization of semiquinones and polymerization through quinones. Thus, autoxidation of alkyl gallates in alkaline medium leads to ellagate, whereas quinone polymerization occurs during the autoxidation of $(+)$-catechin. A similar polymer was isolated from Acacia catechu heartwood, and it was suggested that it arises as a result of aerobic oxidation in the heartwood tissue. Some further examples of natural phenolic polymers were presented. It was pointed out that aerobic oxidation of eatechins and other flavan-3-ols appears to account for the origin of some of the condensed tannins, particularly in tan barks. Brief mention was made of the anti-fungal and anti-viral protective properties of tannin, which, especially in stembark, may have contributed to the successful evolution of existing forest trees.

Prof. H. S. Olcott (University of California, Berkeley) spoke about the effects of tocopherols in autoxidizing systems. He also directed attention to the vitamin $E$ activity of selenium compounds and wondered whether their antioxidant activity is based on the formation of oxides similar to those described by Dr. Bateman in the case of sulphur compounds. The antagonizing effect of free fatty acids on the activity of phenolic antioxidants was contrasted with the synorgistic effect of simple amines. With bases such as tri-iso-octylamine, more effective synergism was demonstrable with phenolic antioxidwnts that possess a hydrogen atom adjacent to the phenolic group ( $\gamma$-tocopherol, butylated hydroxy anisole) than with those in which these positions are substituted ( $\alpha$-tocopherol, butylated hydroxy toluene). Relatively large amounts of aliphatic amines (in the order of 1-2 per cent) act themselves as antioxidants in lard and exhibit an anomalous dependence on temperature in their efficiency. In the discussion of this paper Dr. Barnard (British Rubber Producers' Research Association, Welwyn) confirmed that selenium compounds were found to be more efficient antioxidants than the corresponding sulphur compounds. Dr. E. M. Learmonth (British Soya Products, Puckeridge) directed attention to the instability of purified soybean oil and the synergistic effects of phospholipids. Dr. Uri said that some of the anomalous concentration effects could be explained by the disappearance of the antioxidant by the 'forbidden route' of a chein reaction, and some of the anomalous temperature effects could conceivably be due to chelating of amines with traces of metal porphyrins. Dr. Bateman proposed that certain synergistic effects should be ascribed to a high reactivity of one antioxidant towards $R \mathrm{O}_{2}$. radicals and $\mathbf{a}$ high reactivity of the other towards $R \cdot$ radicals.

Presenting his paper on autoxidation in relation to food, Dr. C. H. Lea distinguished between the major autoxidixible constituent, that is, unsaturated fatty acids, and a wide variety of minor but almost equally important substances; their oxidation is frequently coupled with that of the fats. Dr. Lea went on to survey the main primary and secondary products as well as their effects on flavour, nutritive value and possible toxicity. Finally, a number of factors which influence autoxidative reactions were discussed, including both pro-oxidant and anti-oxidant effects in 
general, the action of hæm protein catalysts and lipoxidase, and finally the variable effects of aseorbic acid which, depending on the environment, acts either as pro-oxidant or as anti-oxidant.

The last paper of the meeting was that of Dr. L. A. O'Neill (Paint Research Station, Teddington) on problems of autoxidation in the paint industry. $\mathrm{He}$ pointed out that paints which depend on the ausoxidation of a drying oil or a polymeric derivative thereof, such as an oil-modified alkyd resin, are still the most widely used class. The oxidation involves the formation of $-\mathrm{C}-\mathrm{C}-$ polymers via conjugated per- oxides. Emphasis was placed on the interesting distinction between the catalytic action of cobalt compounds with oxidation spieading from the surface into the body of the medium and that of lead compounds with uniform oxidation of the bulk of the oil. Oximes are used as temporary antioxidants for sturage in containers and afterwards lost from the surface film. Mercaptans prevent the oxidation from proceeding towards degradation. The nature of 'yellowing' on prolonged ageing, which is accelerated by ammonia and other bases in the atmosphere, is not yet fully undersíood.
N. URI

\section{AVERAGE WATER VAPOUR CONTENT OF THE AIR}

$I^{\mathrm{N}}$ a recent memoir issued by the Meteorological Office, J. K. Bannon and L. P. Steele outline the first use of humidity observations made with radiosondes to describe, on a nearly world scale, the variation with height of the mean water vapour content of the atmosphere (Air Ministry: Meteorological Office. Geophysical Memoirs No. 102: Average Water-Vapour Content of the Air. Pp. ii +38. (M.O. 631 b). London: H.M. Stationery Office, $1960.8 s .6 d$. net). Previous work of this nature was necessarily carried out by extrapolating from surface values with empirical formulæ, deduced from mountain observations, for the rate of decrease with height.

The data, which are for the period 19511955, are presented in the form of charts for January, April, July and October of isopleths of the average weight of water vapour in decigrams above 1 square centimetre at the Earth's surface and the isobaric surfaces for $850 \mathrm{mb}$. (about $1.5 \mathrm{~km}$. height), $700 \mathrm{mb}$. (about $3 \mathrm{~km}$.) and $500 \mathrm{mb}$. (about $5.5 \mathrm{~km}$.). Surface pressure is about $1,000 \mathrm{mb}$.

The authors necessarily devote much space to a detailed discussion of the observations and of the difficult problems involved in reconciling observations made with different types of radiosonde and allowing for instrumental defects such as lag.
The most obvious feature of the charts at any one level is that water content is highest over the equatorial rain forests and India in the summer monsoon, and least over the polar regions. Values are notably low for the latitude over the Sahara, where they are about the same as over Europe, but the other great desert areas are not very prominent in the run of the isopleths. Comparing the charts at different levels, it is clear that at all seasons, and over all areas on the average, half the water vapour content of the atmosphere is below $850 \mathrm{mb}$., far below the corresponding level for air at $500 \mathrm{mb}$. The highest value is over India in the summer monsoon, when the atmosphere holds 64 decigrams of precipitable water. Above the equatorial belt there is about 50 decigrams. Over the British Isles in winter the amount is about 11 decigrams and in summer 24 decigrams.

The charts permit rough calculations to be made of the ratio of precipitation to storage of water vapour in the atmosphere. Over the Earth as a whole the store is equivalent to nine days rainfall, but over wet cool regions such as the Bricish Isles in winter the average store is equivalent to only about three days rainfall. Such differences in the storage ratio reflect differences in the manner of production of rain, which is mainly by convection of 'local' water vapour over the hot regions and by advection from over the oceans in cool ones.

\section{FREEZING AND DRYING OF BIOLOGICAL MATERIALS}

$I^{N}$ recent years much has been said about the rift between science and the humanities. Nevertheless, the majority of scientists are interested in history or literature, music, drama or art, while most students of the humanities use products of science and notice the impact of others on the world at large. The widening gap between different scientific disciplines may be more serious. A stage is approaching when biologists and physicists will no longer understand one another whether they try to communicate orally or by writing. Several attempts to bridge this gap have already been made by those who investigate the effects of low temperatures and of desiccation on living cells and tissues and on their products.
The publication "Freezing and Drying of Biological Materials" is based on a conference held at the New York Academy of Sciences in October 1959*. It forms a valuable sequel to two colloquia organized by the Institute of Biology in 1951 and 1958, to the discussion at the Royal Society in 1957, and to Prof. D. Keilin's Leeuwenhoek Lecture in 1958. This is not a book for beginners. Dr. H. T. Meryman is overoptimistic in saying that a stage of simplification has been reached in cryobiology. Prof. B. Luyet is nearer the truth in thinking that the biologist who works at low temperatures is in "a chaos of disequi-

* Annals of the New York Academy of Sciences. Vol. 85, Article 2 Freezing and Drying of Biological Materials. By Harold $\mathbf{T}$. Meryman and 12 other authors. Pp. 501-734. (New York: New York Aeademy of Sciences, 1960 . 